\title{
Charm Physics at BESIII
}

\section{Xin SHI ${ }^{* \dagger}$}

Institute of High Energy Physics, Beijing 100049, People's Republic of China

E-mail: shixineihep.ac.cn

BESIII collected the world largest data samples of 2.93, 0.482 and $0.567 \mathrm{fb}^{-1}$ data at 3.773 , 4.009 and $4.6 \mathrm{GeV}$, respectively. Based on these data samples, BESIII perform some analyses of $D^{0(+)}, D_{s}^{+}$and $\Lambda_{c}^{+}$, which are important to understand the weak decay machanism of charmed mesons and baryons. The leptonic decays of $D^{+} \rightarrow \tau^{+} v_{\tau}$ and $D_{s}^{+} \rightarrow \ell^{+} v_{\ell}$ are measured. The dynamics of $D^{+} \rightarrow \bar{K}^{0} e^{+} v_{e}, \pi^{0} e^{+} v_{e}$ and $K^{-} \pi^{+} e^{+} v_{e}$ are studied, and the parameters of the form factors and CKM matrix elements $\left|V_{c s(d)}\right|$ are extracted. In addition, the branching fractions for $D^{+} \rightarrow \bar{K}^{0} \mu^{+} v_{\mu}, \bar{K}^{0} e^{+} v_{e}, D_{s}^{+} \rightarrow \eta^{(\prime)} e^{+} v_{e}$ are also provided. The amplitude analysis of $D^{0} \rightarrow K^{-} \pi^{+} \pi^{+} \pi^{-}$is performed. The asymmetries of $D^{+} \rightarrow K_{S / L} K^{+}\left(\pi^{0}\right)$ and $D^{0} \rightarrow K_{S / L} \pi^{0}\left(\pi^{0}\right)$ decays are measured. The branching fractions for $D^{+} \rightarrow 2 K_{S} K^{+}, 2 K_{S} \pi^{+}, D^{0} \rightarrow 2 K_{S}, 3 K_{S}$ as well as $D^{0(+)} \rightarrow$ other 14 channels of PP final states are determined. Finally, the measurement of the Singly-Cabibbo-suppressed decays $\Lambda_{c}^{+} \rightarrow p \pi^{+} \pi^{-}, p K^{+} K^{-}$, the Cabibbo-favored decays of $\Lambda_{c}^{+} \rightarrow \Sigma^{-} \pi^{+} \pi^{+}\left(\pi^{0}\right)$ have been obtained.

XXV International Workshop on Deep-Inelastic Scattering and Related Subjects

3-7 April 2017

University of Birmingham, $U K$

\footnotetext{
* Speaker.

${ }^{\dagger}$ On behalf of BESIII collaboration
} 


\section{Overview}

The BEPCII (Beijing Electron-Positron Collider) is located at the Institute of High Energy Physics in Beijing China. It's beam energy is $1.0-2.3 \mathrm{GeV}$ with optimum energy at $1.89 \mathrm{GeV}$. It has recently achieved it's design luminosity of $1.00 \times 10^{33} \mathrm{~cm}^{-2} \mathrm{~s}^{-1}$. The BESIII [1] detector stated to take data from 2009.

Leptonic and semileptonic decays of charmed mesons $\left(D^{0}, D^{+}, D_{s}^{+}\right.$, and $\left.\Lambda_{c}^{+}\right)$provide an ideal window to explore weak and strong effects. For $D$ leptonic decays: the measurement of $f_{D_{(s)}^{+}}$ and $f^{K(\pi)}$ can be used to better calibrate LQCD, while the $\left|V_{c s(d)}\right|$ will improve the test on CMK unitarity. For $D$ hadronic decays: the $D^{0} \bar{D}^{0}$ mixing parameters are used for $\mathrm{CP}$ violation and the strong phase in $D^{0}$ decays served as constraint on $\gamma / \phi_{3}$ measurement in $B$ decays. The study of rare $D$ decays aims to explore new physics beyond the SM. In addition, the absolute branching fractions of $\Lambda_{c}^{+}$using near threshold data will be the first measurement in the past 40 years.

\section{2. $D$ leptonic decays}

In the Standard Model, the amplitude of $D_{(s)}^{+}$leptonic decays can be described as:

$$
\Gamma\left(D_{(s)}^{+} \rightarrow \ell^{+} v_{\ell}\right)=\frac{G_{F}^{2} f_{D_{(s)+}}^{2}}{8 \pi}\left|V_{c d(s)}\right|^{2} m_{\ell}^{2} m_{D_{(s)}^{+}}\left(1-\frac{m_{\ell}^{2}}{m_{D_{(s)}^{+}}^{2}}\right)^{2}
$$

which serves as a bridge to precisely measure the decay constant $f_{D(s)^{+}}$with input $\left|V_{c d(s)}\right|^{C M K \text { fitter }}$ and CKM matrix element $\left|V_{c d(s)}\right|$ with input $f_{D(s)^{+}}^{L Q C D}$.

To measure the $B\left(D^{+} \rightarrow \mu^{+} v\right)$, the $D^{-}$mesons are tagged by their beam-energy-constrained mass $M_{B C}$ :

$$
M_{B C}=\sqrt{E_{\text {beam }}^{2}-\left|\vec{p}_{m K n \pi}\right|^{2}}
$$

where $m$ and $n(m=0,1,2 ; n=0,1,2,3$, or 4$)$ denotes the numbers of kaons and pions in the tagged $D^{-}$decay mode. The beam-energy-constrained mass distributions for different $\mathrm{D}$-tagged modes is shown in Fig. 1 and the total of $1703054 \pm 3405$ tagged $D^{-}$mesons are found [2]. The branching fraction is measured as $B\left(D^{+} \rightarrow \mu^{+} v\right)=(3.71 \pm 0.19 \pm 0.06) \times 10^{-4}$. Then the pseudoscalar decay constant $f_{D^{+}}$can be inferred as $f_{D^{+}}=(203.2 \pm 5.3 \pm 1.8) \mathrm{MeV}$. The quark mixing matrix element can be also extracted $\left|V_{c d}\right|=0.2210 \pm 0.0058 \pm 0.0047$.

The decay channel(s) of $D^{0+} \rightarrow \pi^{-(0)} l^{+} v$ can be used to study the lepton universality (LU), which is defined as:

$$
R_{L U}^{0(+)}=\frac{B\left(D^{0(+)} \rightarrow \pi^{-(0)} \mu^{+} v\right)}{B\left(D^{0(+)} \rightarrow \pi^{-(0)} e^{+} v\right)}
$$

Using the BFs from PDG16 [3], one can get: $R_{L U}^{0}=0.82 \pm 0.08(\sim 2.0 \sigma)$ in which the large error in $B\left(D^{0} \rightarrow \pi^{-} \mu^{+} v\right)$ and no measure of $B\left(D^{+} \rightarrow \pi^{0} \mu+v\right)$. Precision measurements are desired.

BESIII measures $B\left(D^{0} \rightarrow \pi^{-} \mu^{+} v\right)=(0.267 \pm 0.007 \pm 0.007) \%$ agrees with PDG and with better precision. In additon, the $B\left(D^{+} \rightarrow \pi^{0} \mu^{+} v\right)=(0.342 \pm 0.011 \pm 0.010) \%$ is measured for the 

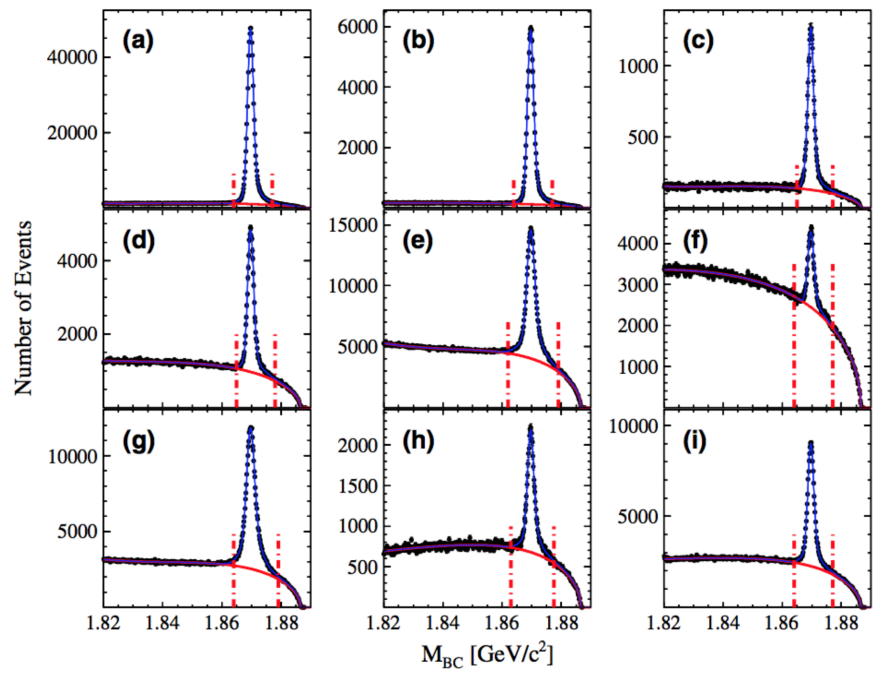

Figure 1: The beam-energy-constrained mass distributions for different D-tagged modes.

first time. Using these updated input, one can get $R_{L U}^{0}=0.918 \pm 0.036$ and $R_{L U}^{+}=0.921 \pm 0.045$ respectively, which agrees with the expectation in 1.5(1.1) $\sigma$.

The isospin symmetry (IS) which is defined as $R_{I S}^{\ell}=\frac{\Gamma\left(D^{0} \rightarrow \pi^{-} \ell^{+} v\right)}{2 \Gamma\left(D^{+} \rightarrow \pi^{0} \ell^{+} v\right)}$ can be also measured as $R_{I S}^{e}=1.03 \pm 0.03 \pm 0.02$ and $R_{I S}^{\mu}=0.990 \pm 0.054$ respectively, which agrees with IS prediction within uncertainty.

In Standard Model the $D^{0} \bar{D}^{0}$ mixing, $C P$ violation and rare decay of charm are small. $D^{0} \bar{D}^{0}$ mixing parameters are: $x \simeq y \simeq 10^{-3}$ which leads to $r_{D}=\left[x^{2}+y^{2}\right] / 2 \simeq 10^{-6}$. The $\mathrm{CP}$ violation asymmetries is in the order of $\sim 10^{-3}$, and rare decays is $\leq 10^{-6}$. On BESIII, the fitting of 6 dominant $D^{-}$single tag, where the branching fraction found to be $B\left(D^{+} \rightarrow D^{0} e^{+} v\right)<8.7 \times 10^{-5}$.

\section{3. $D$ hadronic decays}

Studies of the singly Cabibbo-suppressed (CSC) decays is limited by data set and background. It helps to better understanding of SU(3) symmetry breaking and CP violation, also improve the theory calculation. Using the double tag method, as shown in Fig. 2. This work is published in Ref. [4].

The amplitude analysis of $D^{0} \rightarrow K^{-} \pi^{+} \pi^{+} \pi^{-}$helps to determine the absolute BF, strong phase, benefit $\gamma / \phi_{3}$. Previous analyses only from MarkIII and E691.

The study of the hadronic decays of charmed $D$ mesons is of great significance in the study of the strong and weak interactions in $D$ decays. For example, the analysis on $D \rightarrow P P$ modes will provide materials for the study of $\mathrm{SU}(3)$ breaking effect, while the observation of $\mathrm{CP}$ violation in $\mathrm{D}$ decay is commonly believed to be indications of new physics. Also, the $D^{0} \rightarrow K^{-} \pi^{+}$is an important normalization mode.

Most of the D decays have been studied by CLEO in 2010, other measurements come from Belle, BaBar and CDF, etc. Some of the branching fractions (BFs) are not well established. With 

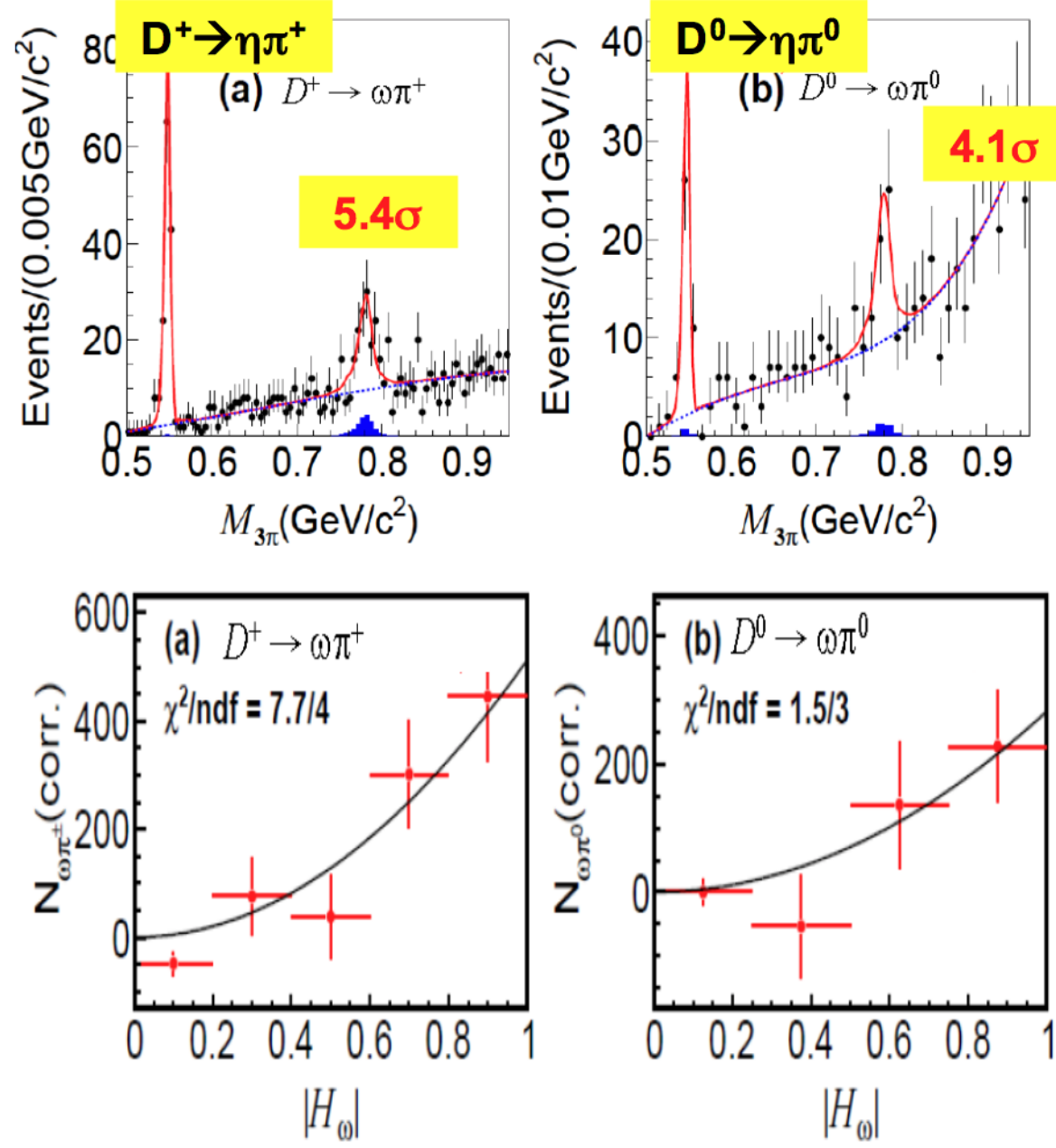

Figure 2: Fitting results of $D$

the $2.93 \mathrm{fb}^{-1}$ data taken at $3.773 \mathrm{GeV}$ within BESIII, the results will help to improve these measurements, the preliminary fitting results are shown in Fig. 3.

\section{4. $\Lambda_{c}^{+}$decays}

Although the $\Lambda_{c}^{+}$was observed in 1979, before 2014, all decays of $\Lambda_{c}^{+}$are measured relative to $\Lambda_{c}^{+} \rightarrow p K^{-} \pi^{+}$, which suffer large error of $25 \%$. There was no absolute measurement using data produced at $\Lambda_{c}^{+}$pair threshold. The sum of BFs of known decays $\Lambda_{c}^{+}$is only about $60 \%$. This situation was improved till 2014, when Belle reported improved measurement of $B\left[\Lambda_{c}^{+} \rightarrow p K^{-} \pi^{+}\right]$ with a precision of $\sim 5 \%$. In general, the systematic studies of $\Lambda_{c}^{+}$, search for new decays, absolute BF measurements are important to fully explore the $\Lambda_{c}^{+}$decay mechanisms.

With BESIII data, some BFs of $\Lambda_{c}^{+}$decays are significantly improved [5], where the single tags are around 15000 as shown in Fig. 4. The first absolute BFs of $\Lambda_{c}^{+} \rightarrow \Lambda \ell^{+} v$ are measured: 

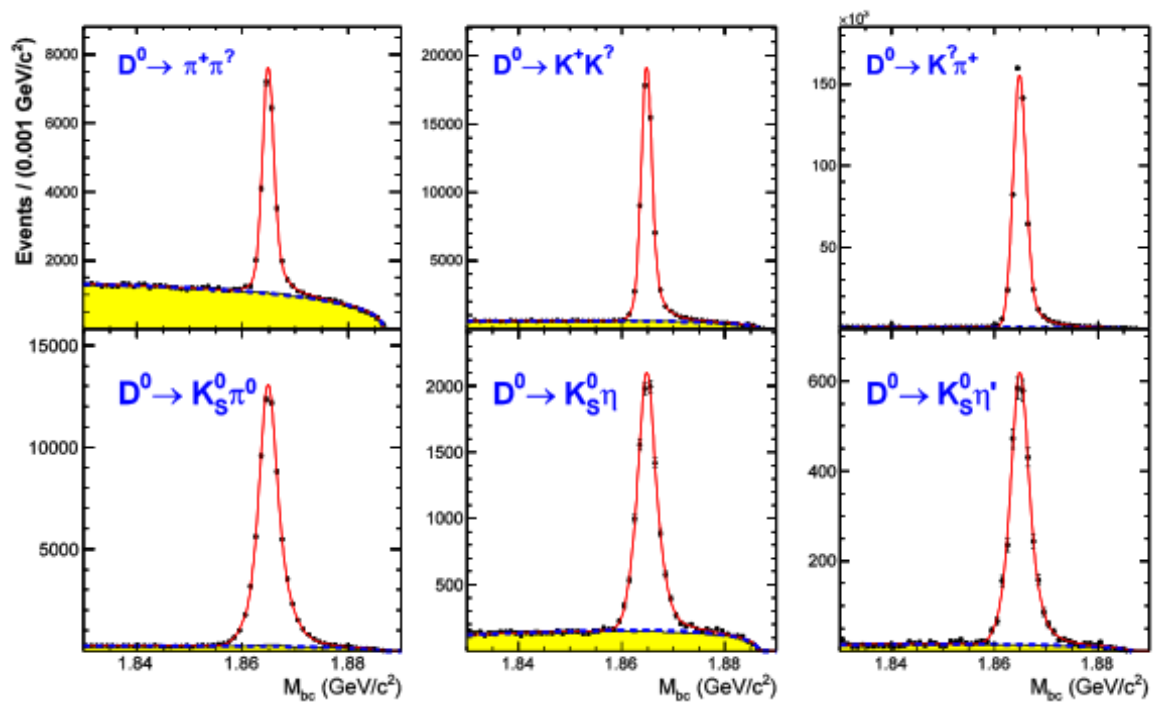

Figure 3: Fitting results of $D \rightarrow P P$

$B\left[\Lambda_{c}^{+} \rightarrow \Lambda e^{+} v_{e}\right]=(3.63 \pm 0.38 \pm 0.20) \%[6]$ and $B\left[\Lambda_{c}^{+} \rightarrow \Lambda \mu^{+} v_{\mu}\right]=(3.49 \pm 0.46 \pm 0.26) \%$ [7] The ratio can be calculated: $\Gamma\left[\Lambda_{c}^{+} \rightarrow \Lambda \mu^{+} v_{\mu}\right] / \Gamma\left[\Lambda_{c}^{+} \rightarrow \Lambda e^{+} v_{e}\right]=0.96 \pm 0.16 \pm 0.04$.

The singly Cabibbo-suppressed (SCS) decays $\Lambda_{c}^{+} \rightarrow p K^{+} K^{-}$and $p \pi^{+} \pi^{-}$helps to distinguish predictions from different theoretical models and understand contributions from factorable effects. BESIII obtain ratios of branching fractions: $\left[B\left(\Lambda_{c}^{+} \rightarrow p \pi^{+} \pi^{-}\right) / B\left(\Lambda_{c}^{+} \rightarrow p K^{-} \pi^{+}\right)\right]=$ $(6.70 \pm 0.48 \pm 0.25) \%[8]$.

BESIII also made the first observation of $\Lambda_{c}^{+} \rightarrow \Sigma^{-} \pi^{+} \pi^{+} \pi^{0}$ with $B\left[\Lambda_{c}^{+} \rightarrow \Sigma^{-} \pi^{+} \pi^{+} \pi^{0}\right]=$ $(2.11 \pm 0.33) \%$, where the errors are statistical only. These sources of the systematic errors arise mainly from the systematic uncertainties in PID, tracking, $\pi^{0}$ efficiency, fitting, MC statistics and number of $\bar{\Lambda}_{c}^{-}$tags. The total systematic errors are estimated to be about 5\%. The measured branching fraction for $\Lambda_{c}^{+} \rightarrow \Sigma^{-} \pi^{+} \pi^{+}=(1.81 \pm 0.17) \%$ is consistent with and more precise than PDG2015.

\section{Summary}

With 2.93, 0.482, $0.567 \mathrm{fb}^{-1}$ data taken at 3.773, 4.009 and $4.6 \mathrm{GeV}$, BESIII have studied leptonic related and hadronic decays of D, first measurement of the absolute BFs of $\Lambda_{c}^{+}$, improved measurements of decay constant $f_{D^{+}}$and form factor $f_{+}^{D \rightarrow K(\pi)}\left(q^{2}\right)$, which are important to test and calibrate LQCD calculations. It also improved the measurements of CKM matrix element $\left|V_{c s(d)}\right|$, which are important to test the CKM matrix unitarity. In addition, about $3 \mathrm{fb}^{-1}$ data at 4.18 $\mathrm{GeV}$ has been taken in 2016, measurement of $f_{D_{s}^{+}}$and $\mid V_{c s}$ by $D_{s}^{+} \rightarrow l^{+} v$, form factor studies of $D_{s}^{+} \rightarrow \eta(\prime) e^{+} v$ are expected in the near future. 


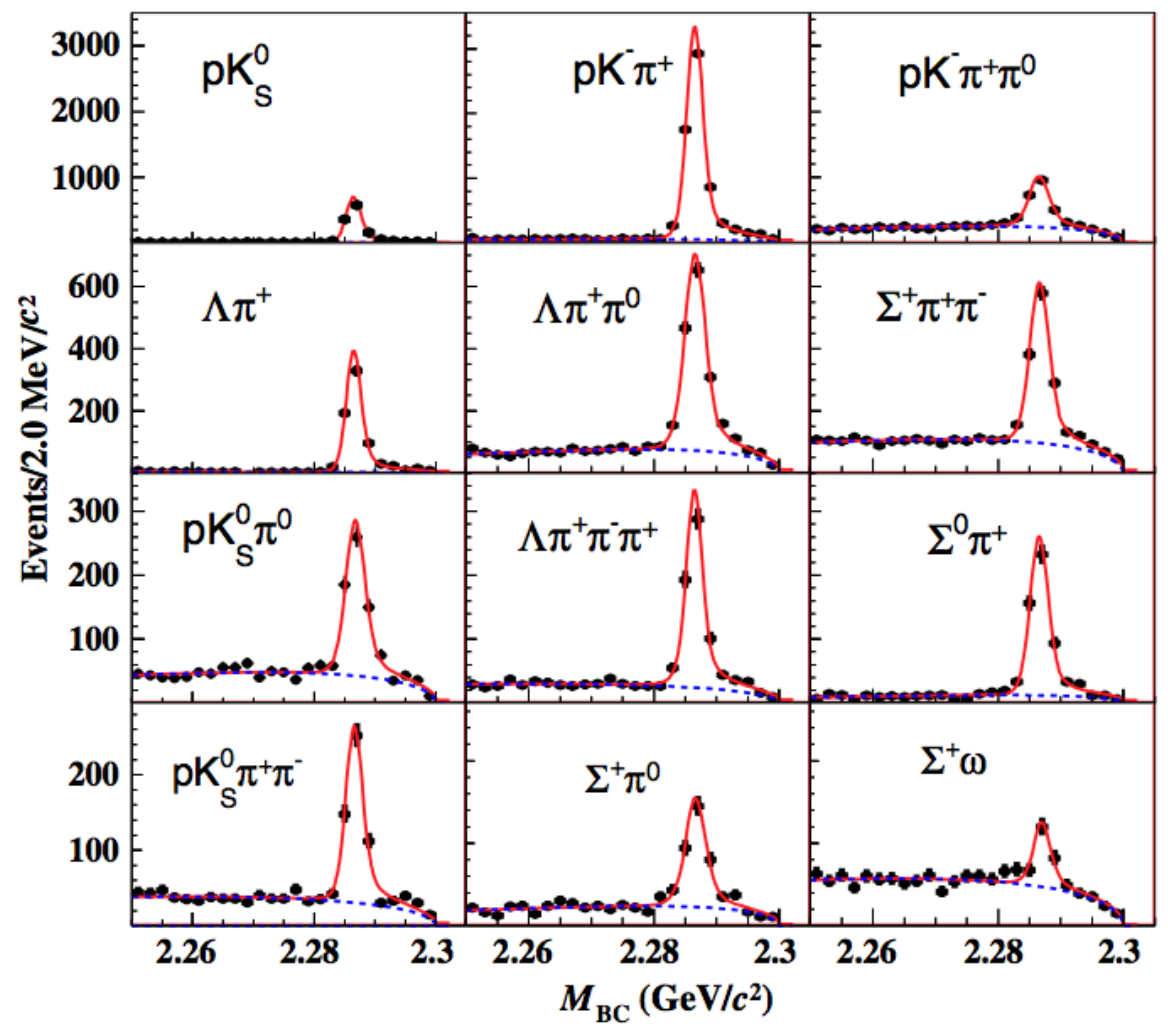

Figure 4: Fitting to the ST $M_{B C}$ distributions in data for the different decay modes.

\section{References}

[1] BESIII collaboration, M. Ablikim et al., Design and Construction of the BESIII Detector, Nucl. Instrum. Meth. A614 (2010) 345-399, [0911.4960].

[2] BESIII collaboration, M. Ablikim et al., Precision measurements of $B\left(d^{+} \rightarrow \mu^{+} v_{\mu}\right)$, the pseudoscalar decay constant $f_{D^{+}}$, and the quark mixing matrix element $\left|V_{c d}\right|$, Physical Review $D 89$ (Mar, 2014) .

[3] Particle Data Group collaboration, C. Patrignani et al., Review of Particle Physics, Chin. Phys. C40 (2016) 100001.

[4] BESIII collaboration, M. Ablikim et al., Observation of the singly cabibbo-suppressed decay $D^{+} \rightarrow \omega \pi^{+}$and evidence for $D^{0} \rightarrow \omega \pi^{0}$, Phys. Rev. Lett. 116 (Feb, 2016) 082001.

[5] M. Ablikim et al., Measurements of Absolute Hadronic Branching Fractions of the $\Lambda_{c}^{+}$Baryon, Physical Review Letters 116 (2016) 1-7.

[6] M. Ablikim et al., Measurement of the Absolute Branching Fraction for $\Lambda_{c}^{+} \rightarrow \Lambda e^{+} v_{e}$, Phys. Rev. Lett. 115 (2015) 221805.

[7] Measurement of the absolute branching fraction for $\Lambda_{c}^{+} \rightarrow \Lambda \mu^{+} v_{\mu}$, Physics Letters $B 767$ (2017) $42-$ 47.

[8] M. Ablikim et al., Measurement of Singly Cabibbo Suppressed Decays $\Lambda_{c}^{+} \rightarrow p \pi^{+} \pi^{-}$and $\Lambda_{c}^{+} \rightarrow p K^{+} K^{-}$, Physical Review Letters 117 (2016) 1-7. 